\title{
Podiatry intervention versus usual care to prevent falls in care homes: pilot randomised controlled trial (the PIRFECT study)
}

Gavin Wylie ${ }^{1 *}$, Hylton B. Menz ${ }^{2}$, Sarah McFarlane ${ }^{3}$, Simon Ogston ${ }^{4}$, Frank Sullivan ${ }^{5}$, Brian Williams ${ }^{6}, Z_{\text {Zoe }}$ Young $^{7}$ and Jacqui Morris ${ }^{8}$

\begin{abstract}
Background: Common foot problems are independent risk factors for falls in older people. There is evidence that podiatry can prevent falls in community-dwelling populations. The feasibility of implementing a podiatry intervention and trial in the care home population is unknown. To inform a potential future definitive trial, we performed a pilot randomised controlled trial to assess: (i) the feasibility of a trial of a podiatry intervention to reduce care home falls, and (ii) the potential direction and magnitude of the effect of the intervention in terms of number of falls in care home residents.

Methods: Informed by Medical Research Council guidance on developing and evaluating complex interventions, we conducted a single blind, pilot randomised controlled trial in six care homes in the East of Scotland. Participants were randomised to either: (i) a three month podiatry intervention comprising core podiatry care, foot and ankle exercises, orthoses and footwear provision or (ii) usual care. Falls-related outcomes (number of falls, time to first fall) and feasibility-related outcomes (recruitment, retention, adherence, data collection rates) were collected. Secondary outcomes included: generic health status, balance, mobility, falls efficacy, and ankle joint strength.
\end{abstract}

Results: 474 care home residents were screened. 43 (9.1\%) participants were recruited: 23 to the intervention, 20 to control. Nine (21\%) participants were lost to follow-up due to declining health or death. It was feasible to deliver the trial elements in the care home setting. 35\% of participants completed the exercise programme. $48 \%$ reported using the orthoses 'all or most of the time'. Completion rates of the outcome measures were between $93 \%$ and $100 \%$. No adverse events were reported. At the nine month follow-up period, the intervention group per-person fall rate was 0.77 falls vs. 0.83 falls in the control group.

Conclusions: A podiatry intervention to reduce falls can be delivered to care home residents within a pilot randomised controlled trial of the intervention. Although not powered to determine effectiveness, these preliminary data provide justification for a larger trial, incorporating a full process evaluation, to determine whether this intervention can significantly reduce falls in this high-risk population.

Trial registration: ClinicalTrials.gov identifier: NCT02178527; Date of registration: 17 June 2014.

Keywords: Older people, Falls, Podiatry, Care homes

\footnotetext{
* Correspondence: g.wylie@nhs.net

${ }^{1} \mathrm{NHS}$ Tayside, Ageing and Health, and School of Nursing \& Health Sciences,

University of Dundee, Dundee, UK

Full list of author information is available at the end of the article
} 


\section{Background}

Half of all care home residents fall every year, often several times [1-3]. They are at an increased risk of falls compared to community dwelling older people because they are more frail, and frequently have multiple longterm health problems [4], for which they are taking multiple medications known to impair balance [5]. Across community and care home dwelling older people, falls are one of the most common causes of hip fracture, unplanned hospitalisation and death [6]. Despite the disproportionately high number of falls in care homes, a Cochrane systematic review found the evidence supporting effectiveness of interventions to reduce falls in care homes to be equivocal [7]. One recent feasibility randomised controlled trial (RCT) has shown that a risk assessment intervention to reduce care home falls is implementable under trial conditions, but it has not yet been tested in a full scale RCT [8].

Falls result from complex interplay between environmental hazards and physiological risk factors such as impaired muscle strength, balance and gait [5]. Chronic disease that leads to impairments in sensory, neurological, cognitive and musculoskeletal functioning also increases the risk of falls [9]. More recently, foot problems, which are common in older people $[10,11]$, have been shown to be a contributing factor to falls $[12,13]$. Foot and ankle risk factors for falls are: reduced ankle joint range of motion, hallux valgus deformity (bunion), decreased toe plantarflexor strength, increased shoe heel height, inadequate shoe fixation (absence of strap or lace or other retaining medium) and reduced shoe-sole contact area $[12,14,15]$. Furthermore, recent reviews suggest that foot and ankle exercises (particularly toe exercises), and footwear interventions (shoes or foot orthoses) may improve falls-risk related outcomes such as static and dynamic balance ability, ankle flexibility and lateral stability $[16,17]$.

Given the demonstrable link between feet, footwear and falls, an inexpensive and simple to implement multifaceted podiatry intervention has been developed and studied within an Australian community dwelling cohort of older people in a large RCT [18]. This work demonstrated that the intervention group experienced 36\% fewer falls compared to those in the control group, and significantly reduced the incidence of falls compared to usual care (incidence rate ratio 0.64, 95\%; CI 0.45 to 0.91). More recently, a cohort-RCT using a similar podiatry intervention conducted with a United Kingdom (UK) community dwelling population demonstrated a reduction in the incidence rate of falls in the intervention group (incidence rate ratio $0.88,95 \%$ CI 0.73 to $1.05)$ and a reduction in the proportion of multiple fallers $(27.6 \%$ vs $34.6 \%)$ [19]. The podiatry intervention holds promise as a falls-reduction strategy. However, the results of falls trials conducted in community dwelling older people cannot be directly applied to those living in care home settings who are more frail, prone to sudden declines in health, and more cognitively and functionally impaired than those living in their own homes. Therefore, exploring the feasibility and potential effectiveness of the intervention in the care home setting - the population most at risk of falls - is warranted.

Challenges in delivering interventions and conducting trials in care homes include recruitment, engaging care home staff, and ensuring that the intervention is feasible and deliverable $[20,21]$. Given these uncertainties, we conducted a two-stage study that was informed by the Medical Research Council (MRC) guidance on complex intervention development [22] (the PIRFECT study Podiatry Intervention to Reduce Falls in Elderly Care Trial). In the first stage we remodelled the existing multifaceted podiatry intervention that was designed for community-dwelling older people [18] to ensure that it was feasible and acceptable in the care home setting [23]. We also refined recruitment processes and determined appropriate outcomes [24]. The second stage of the study, which is reported here, was a pilot RCT of the remodelled multifaceted podiatry intervention.

We aimed to establish the feasibility of a future phase III multicentre RCT designed to establish the clinical effectiveness of the remodelled multifaceted podiatry intervention. The objectives of this pilot study were to examine the recruitment and retention rate, adherence, the quality of the outcome data, and to estimate the potential effect size of the intervention on the number of falls. We anticipated that this approach would inform decision-making regarding progression to a large-scale definitive phase III RCT.

\section{Methods \\ Design}

The study was a two arm, parallel group pilot randomised controlled trial with outcome assessments at baseline (T1), and subsequent blinded outcome assessment at the end of the intervention (T2) and at 3 months (T3) and 6 months (T4) after the end of the intervention.

\section{Setting}

Six care homes in the East of Scotland. Four care homes were privately owned, one was operated by a charitable trust, and one was state-run by the local authority. The capacity of each care home ranged from 24 to 55 residents. Each of the privately owned care homes always had at least one registered nurse on duty, with the remaining staff being care assistants. The state-run and charitable trust care homes employed no registered nurse (although had regular visits from UK National Health Service nurses), and were staffed entirely by care assistants. Our 
initial approach to care homes was through a number of routes: directly to the care home, through regulators (the Scottish Care Inspectorate), and via UK National Health Service (NHS) falls service co-ordinators. We met with care home managers who expressed interest to explain in full what was involved in participating in the study. Those who wanted to take part then provided written permission to conduct the study within their homes. The care homes that were recruited were representative of normal UK care home provision and typical of care homes that would be invited to take part in a future multicentre RCT.

\section{Participants and recruitment}

The East of Scotland Research Ethics Service granted ethical approval for the study (reference 12/ES/0088). Care home staff identified and initially approached residents who met the initial inclusion criteria on behalf of the research team. A member of the research team then approached interested residents, to provide further information and answer any questions. A researcher then screened the potential participant using the inclusion/ exclusion criteria before obtaining written informed consent.

Participants were eligible for recruitment if they fulfilled the following inclusion criteria: (i) $>65$ years old and permanently living in a care home for older people; (ii) one or more falls in the previous year (in order to target those most at risk of a fall [25]); (iii) a foot problem within the scope of practice of a UK trained podiatrist (defined by the UK Health and Care Professions Council [26]). Residents were excluded if they were: (i) terminally unwell or too frail to be included; (ii) only able to mobilise with the use of wheelchair; (iii) temporary residents; (iv) unable to provide informed consent; (v) lower limb amputees.

We used the mini-mental-state exam (MMSE) [27] to guide our assessment of capacity to consent. We used a severe score (0-9) on the MMSE as an indicative threshold for inability to provide informed consent. However, the final decision to recruit, irrespective of MMSE score, was through assessment by the research podiatrist, based on the ability of the prospective participant to explain back to the research podiatrist what was required by their participation in the study. Where there was ambiguity, a decision on whether or not a potential participant should enter the study was based on agreement between two researchers (GW and ZY).

\section{Randomisation and blinding}

After recruitment, but prior to randomisation, baseline assessments were completed by one of 2 research podiatrists (GW or ZY). Participants were then randomly assigned to the usual care control group or the intervention group. Randomisation was conducted via a concealed, web-based randomisation service provided by UK Clinical Research Collaboration (UKCRC) registered Tayside Clinical Trials Unit at the University of Dundee. Minimisation was employed to ensure balance between the two groups on the following falls-related risk factors: age, gender, presence of polypharmacy ( $>4$ concurrent medications) and the presence of psychotropic medication. Follow up measures were conducted by a rater who was a physiotherapist of 20 years experience (SM), trained in the conduct of all measures and who was unaware of participant allocation. Given the nature of the intervention it was not possible to blind participants and care home staff to group allocation, however blinding of the rater was maintained by ensuring that access to research records indicating group allocation was restricted and by asking participants not to reveal their group allocation to the rater at the outset of each follow up visit.

\section{Outcome measures}

Feasibility outcome data and falls-related outcome data were collected. Feasibility data were collected on recruitment, retention, adherence, and missing data. The primary falls outcome measure was the number of falls in the 9-month trial period. A fall was defined as 'an unexpected event in which the participant comes to rest on the ground, floor or lower level [28]'. Falls recall by care home residents themselves is unreliable [29], therefore data on the number of falls were collected via the accident reporting systems in place within the care homes. All homes kept accident records that recorded both the number of falls and the circumstances surrounding them (where possible), which allowed us to count only falls meeting our definition. We also calculated the time to first fall to assess whether those in the intervention group increased their risk of falls as a result of the intervention. The secondary outcome measures were:

I. Podiatry Objective Clinical Score (POCS) [30]: a clinical measure of current foot problems determined by a podiatrist and scored from 1 (no problems) to 5 (gross problems);

II. Berg Balance Scale (BBS) [31]: a measure of balance function in older people and scored from 0 to 56 where a higher score indicates lower falls risk;

III.Timed Up and Go Test [32]: a simple assessment of mobility where the participant is timed to rise from a chair, walk $3 \mathrm{~m}$, turn round, walk back to the chair and sit down where a time of $30 \mathrm{~s}$ or more to complete the manoeuvre infers that the person is at increased risk of falls;

IV.Barthel Index [33]: a measure of competence in activities of daily living and scored from 0 to 100 where a higher score indicates greater independence; 
V. EQ-5D [34]: a measure of generic health status where a summary index score between zero and one is derived, with one representing the best possible health state;

VI.Nursing Home Falls Self-Efficacy Scale (NHFSS) [35]: a measure of falls efficacy where a higher score indicates less fear of falling

VIIAnkle joint muscle strength: measured via a dynamometer in Newtons [36] whereby a higher score indicates better muscle strength.

Falls outcome data were collected at the following time points: baseline (T1), immediately at the end of the 3 -month intervention (T2), 3 months after the end of the intervention (T3), and finally 6 months after the end of the intervention (T4).

\section{Development of the intervention}

The empirical basis for the intervention was a multifaceted podiatry intervention to reduce falls, previously evaluated in community dwelling Australian and UK populations $[18,19]$. Prior to conducting the study described in the present paper, the intervention was delivered by the research podiatrist in an acceptability study to care home residents $(n=8)$ and care home staff $(n=5)$ from care homes not subsequently recruited to the pilot RCT. Feedback received from care home residents and staff, as well as experience gained by the research podiatrist informed the remodelling of the intervention and its delivery. Briefly, the process was as follows. Staff and residents participated in a 3-month acceptability-testing phase of the initial intervention, and feasibility test of the recruitment strategy. At the conclusion of testing, semi-structured interviews were conducted to assess the acceptability and perceptions of the intervention. Based on these findings, the intervention and its delivery were refined so as to be acceptable and feasible in the care home setting. Furthermore, the research podiatrist worked closely with the care home staff to develop suitable training in order to facilitate delivery of the intervention. Both the remodelling process and feasibility testing of the recruitment strategy are described in more detail elsewhere [23, 24].

\section{Control group}

The control group received core podiatry only, which is defined as routine nail and callus (hard skin) maintenance provided either by the UK National Health Service (NHS) or podiatrists in private practice [30]. Within the geographical area in where the study was conducted (East of Scotland), the NHS provides core podiatry services routinely to all care home residents.

\section{Intervention group}

In addition to the core podiatry that the control group received, the intervention group also received foot orthoses provision, footwear assessment and provision and a course of foot and ankle exercises.

\section{Foot orthoses}

Foot orthoses are reported to improve balance and postural stability by increasing tactile stimulation of the plantar surface of the foot, thereby enhancing afferent somatosensory feedback available to the central nervous system [37]. We supplied and fitted full-length, prefabricated foot orthoses (Formthotics ${ }^{\mathrm{rm}}$, Foot Science International, Christchurch, New Zealand). These are widely available in the UK and mainland Europe. The devices were dual density: the base layer is constructed of high (hard) density thermoformable closed cell polyethylene foam and the top layer constructed of low (soft) density thermoformable closed cell polyethylene foam. The devices were customised for each resident in two ways: firstly, by moulding the devices with a heat gun in order to match the contours of the foot, and secondly by affixing $6 \mathrm{~mm}$ thick Poron $^{\mathrm{Tm}}$ cushioning material (Rogers Corporation, Connecticut) under the forefoot area of the orthotic in order to redistribute pressure away from areas of high pressure (i.e. where plantar lesions such as hyperkeratosis were identified by research podiatrist (GW or ZY) on the plantar aspect of the foot. The cost of supplying orthoses for each resident was $£ 24$ (\$A42; $\$ 31 ; € 28)$. These orthoses were only supplied if the resident was not using orthoses that had been already supplied to them prior to their recruitment to the study.

\section{Footwear assessment and provision}

Residents' current footwear was assessed by the research podiatrist using a validated footwear assessment tool [38]. In cases where residents' footwear was judged inappropriate, residents, family members, and/or relevant care home staff were given a footwear catalogue containing a selection of footwear that, according to the validated footwear assessment tool, met the criteria for optimal footwear (DB Shoes, Northamptonshire, England). Residents were then able to select their preferred footwear from the catalogue, and these were then purchased on behalf of the residents and paid for from the study grant funds. The cost of footwear provision for each resident ranged from $£ 75$ to $£ 89$ (\$A130 to \$A154; $\$ 97$ to $\$ 116$; $€ 87$ to $€ 102$ ).

\section{Foot and ankle exercises}

Table 1 provides details of the exercise programme. The foot and ankle exercises were adapted from those conducted by Spink et al. [18] and Ribeiro et al. [39] to provide a regime that was acceptable within care home routines. The original exercise protocol, for example stated "3 
Table 1 Exercise programme

\begin{tabular}{|c|c|c|c|}
\hline Activity & Description & Frequency & Intensity progression* \\
\hline $\begin{array}{l}\text { Ankle } \\
\text { dorsiflexion } \\
\text { strength* }\end{array}$ & $\begin{array}{l}\text { (i) Sit with knee extended. (ii) Wrap middle of exercise band around foot. (iii) } \\
\text { Grasp ends of bands, and hold at waist, taking up slack. (iv) Push foot down into } \\
\text { the band and return slowly. }\end{array}$ & $3 \times 10$ repetitions & $\begin{array}{l}\text { Increase tension strength } \\
\text { of resistive exercise band }\end{array}$ \\
\hline $\begin{array}{l}\text { Ankle } \\
\text { plantarflexion } \\
\text { strength* }\end{array}$ & $\begin{array}{l}\text { (i) Sit with both knees extended. (ii) Wrap middle of band around the foot of the } \\
\text { ankle to be exercised. (iii) Run the band under opposite foot to hold band in } \\
\text { place. (iv) Grasp ends of bands, and hold at knee height, taking up slack. (v) Lift } \\
\text { foot against the band, hold and slowly return. }\end{array}$ & $3 \times 10$ repetitions & $\begin{array}{l}\text { Increase tension strength } \\
\text { of resistive exercise band }\end{array}$ \\
\hline $\begin{array}{l}\text { Toe } \\
\text { plantarflexion } \\
\text { strength }\end{array}$ & (i) Pick up $25 \mathrm{~mm}$ diameter marbles and place in box & $\begin{array}{l}\text { Pick up } 2 \times 20 \\
\text { marbles for each } \\
\text { foot }\end{array}$ & None \\
\hline
\end{tabular}

*Participants began at an appropriate tension for their current strength capacity. This was determined by finding the tension at which 10 repetitions are possible with full range of motion before fatigue. Once the participant was able to perform 3 sets of 10 repetitions without fatigue, the intensity of the exercise was increased by increasing the resistance of the elastic band. Weekly visits from the research podiatrist allowed assessment of appropriate progression

repetitions of 10 sets". Care home staff and residents found this to be potentially confusing, so this description would be changed to say simply, "30 times". Participants were asked, in collaboration with the care home staff, to complete the exercise component of the intervention 3 times per week (on days of their own choosing) for 3 months. Exercise frequency remained at 3 times per week for the duration of the study. Only the ankle exercises involved progression.

\section{Intervention training}

Care home staff involved in the study participated in a 2-h training session conducted by the research podiatrists to familiarise themselves with the exercise component of the intervention. Intervention training to care home staff and residents was conducted by one of two podiatrists (GW or ZY). Information sheets detailing the exercises as well as a training DVD accessible via the study website, which was active for the duration of the trial (Additional file 1) supported staff in the exercise provision.

\section{Intervention delivery}

The intervention was administered in one of two ways: (i) if, on assessment, the resident demonstrated sufficient cognitive capacity and understanding they were, if they wished, trained to practice the exercises independently, or (ii) if residents did not have sufficient understanding either a family member or a member of care home staff was trained to supervise the exercise programme. Logbooks to record exercise adherence were issued to all participants and placed in a prominent area of each resident's room.

\section{Sample size and statistical analysis}

Since this was a pilot study, a formal sample size calculation was not conducted in advance [40]. We aimed to recruit 40 care home residents to the pilot trial, since it was felt that this would be sufficient to facilitate a later sample size calculation, whilst allowing for participant attrition. The primary falls-related outcome of interest was the number of falls. Cohen's $d$ [41] was also calculated to estimate the effect size of the intervention for the difference in mean falls between the groups at time points T1-T2, T2-T3, and T3-T4. We also used the negative binomial regression model to compare recurrent fall events in both groups [42]. T-tests for the difference in means were used to estimate $p$-values for between group differences in number of falls. Effect size was calculated for the difference in means in the secondary outcomes from T1-T2 and T3-T4. Adherence to the intervention was recorded via exercise logs that were completed either by the care home staff or the resident and analysed descriptively. Finally, median time to first fall was calculated as a safety measure to ensure that those in the intervention group were not at a higher risk of falling [43]. SPSS and Stata were used for the statistical analysis. Analyses were carried out using the intention to treat principle, by multiple imputation of missing at random data.

\section{Results \\ Recruitment}

Figure 1 shows the CONSORT [44] diagram with the flow of participants through the study. Between January 2014 and June 2014, we screened 474 potential participants from six care homes. The main reason for nonrecruitment was inability to provide informed consent. Forty-seven residents $(9.9 \%)$ met the inclusion criteria, agreed to participate and provided informed consent. Four residents dropped out prior to randomisation, thus 43 residents were randomised, representing 9.1\% of the screened population. With the exception of gender, previous stroke, and eye problems, the intervention and control groups were well matched on the majority of baseline demographic characteristics (Table 2). Due to delays in the provision of personalised intervention equipment (orthoses and footwear), the mean number of days in the trial was longer in the intervention 


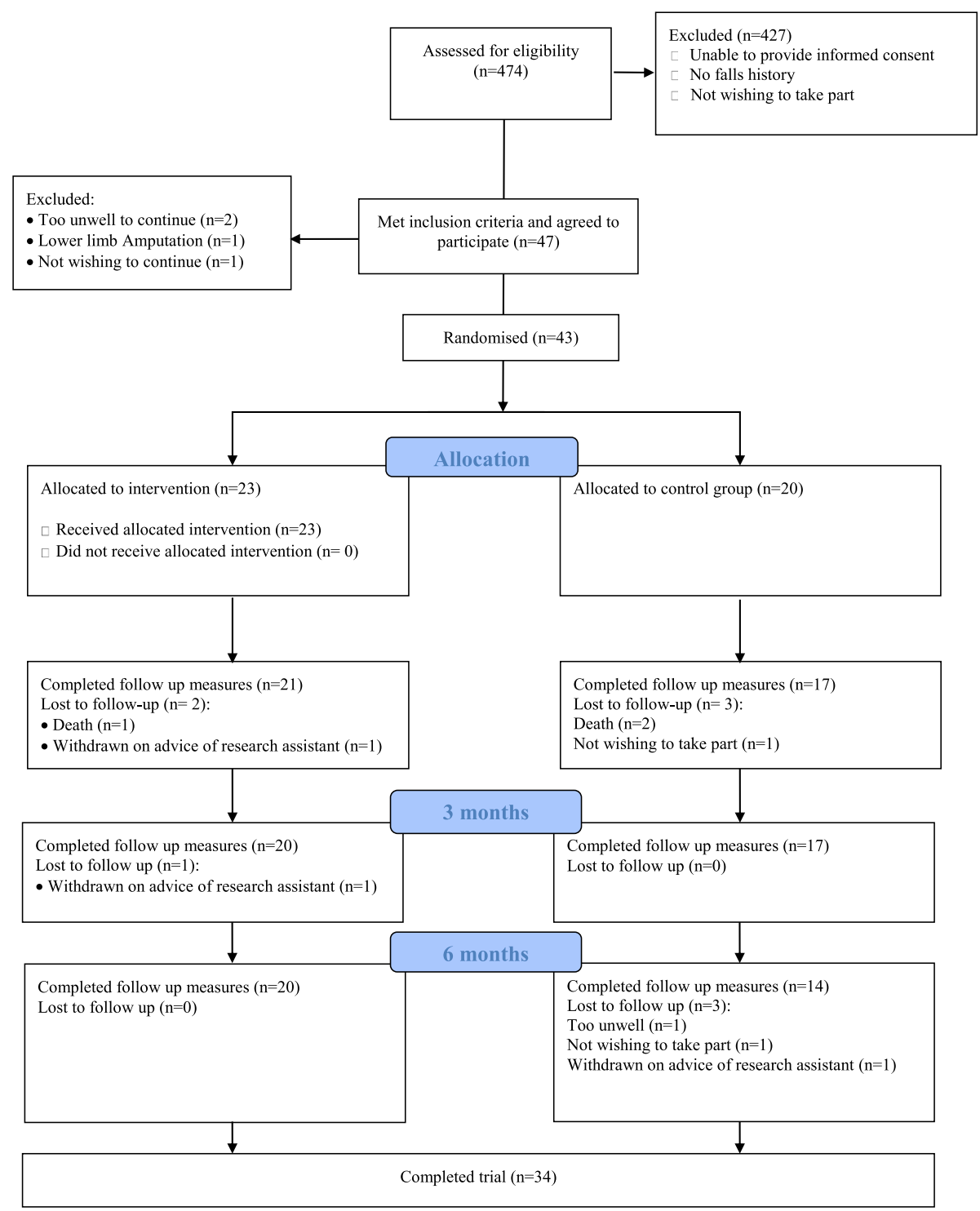

Fig. 1 CONSORT flow of participants through study

group: 257.9 (SD 57.8) compared to the control group: 208.1 (SD 86.5).

\section{Retention}

Between recruitment and randomisation, four residents dropped out. Reasons given: too unwell to continue $(n=2)$, lower limb amputation $(n=1)$, no longer wishing to take part $(n=1)$. Between T1 and T2, 4/23 (17\%) participants in the intervention group dropped out, and 3/17 (18\%) participants dropped out from the control group. Between T2 and T4 one further participant dropped out from the intervention group, and a further three dropped out from the control group. The final trial participant retention rate was $87 \%$ in the intervention group and $70 \%$ in the control group.

\section{Falls-related outcome measures Number of falls}

Table 3 shows the mean number of falls per participant and effect size by time point. 97 falls occurred from baseline (T1) to the end of the 6-month follow up period (T4), with 49 occurring in the intervention group, and 48 in the control group. Of all participants who started the trial, in the intervention group $48 \%(10 / 21)$ experienced at least one fall, and in the control group 12/17 (71\%) experienced at least one fall. 7/21 (33\%) participants in the intervention group had repeat falls, compared with 9/17 (53\%) in the control group. There was one fallrelated fracture in the intervention group (hip) and one fall related fracture in the control group (clavicle). Following multiple imputation to account for missing data on 
Table 2 Baseline participant characteristics

\begin{tabular}{|c|c|c|c|}
\hline Characteristics at screening $^{a}$ & $\begin{array}{l}\text { Podiatry intervention } \\
\text { group } n=23\end{array}$ & Usual care group $n=20$ & Total $n=43$ \\
\hline Male, n (\%) & $3(13 \%)$ & $5(25 \%)$ & $8(19 \%)$ \\
\hline Female, n (\%) & $20(87 \%)$ & $15(75 \%)$ & $35(81 \%)$ \\
\hline Age (years), mean (sd) & $86.9(6.2)$ & $85.9(7.8)$ & $86.4(6.9)$ \\
\hline Body mass index ${ }^{\mathrm{b}}$, mean (sd) & $24.2(4.6)$ & $28.3(5.8)$ & $26.1(5.5)$ \\
\hline MMSE, mean (sd) & $21.2(5.4)$ & $21.0(5.3)$ & $21.2(5.3)$ \\
\hline \multicolumn{4}{|l|}{ Medical History, n (\%) } \\
\hline Diabetes & $2(8.7 \%)$ & $2(10 \%)$ & $4(9.3 \%)$ \\
\hline Peripheral neuropathy & $1(4.3 \%)$ & $0(0 \%)$ & $1(2.3 \%)$ \\
\hline Hypertension & $12(52.2 \%)$ & $8(40 \%)$ & $20(46.5 \%)$ \\
\hline Previous CVA & $8(34.8)$ & $3(15 \%)$ & $11(25.6)$ \\
\hline Peripheral vascular disease & $1(4.3 \%)$ & $2(10 \%)$ & $3(7 \%)$ \\
\hline Angina & $5(21.7 \%)$ & $3(15 \%)$ & $8(18.6 \%)$ \\
\hline Heart failure & $2(8.7 \%)$ & $3(15 \%)$ & $5(11.6)$ \\
\hline$\geq 4$ prescribed medications & $22(95.7 \%)$ & $19(95 \%)$ & $41(95.3)$ \\
\hline \multicolumn{4}{|l|}{ Eye problems (n, \%) } \\
\hline Partially sighted & $4(17.4 \%)$ & $3(15 \%)$ & $7(16.3 \%)$ \\
\hline Registered blind & $0(0 \%)$ & $4(20 \%)$ & $4(9.3 \%)$ \\
\hline Uses walking aids $(n, \%)$ & $18(78.3 \%)$ & $18(90 \%)$ & $36(83.7 \%)$ \\
\hline Currently accessing podiatry services $(n, \%)$ & $23(100 \%)$ & $20(100 \%)$ & $43(100 \%)$ \\
\hline
\end{tabular}

MMSE Mini-Mental State Exam, Higher score better cognition

${ }^{a}$ For continuous variables, means and standard deviations are given. For categorical variables, proportions in each category are given

${ }^{\mathrm{b}}$ weight/height ${ }^{2}$

the nine residents who did not complete the trial, the podiatry intervention group experienced a total mean of 2.3 (SD3.2) falls from $\mathrm{T} 1$ to $\mathrm{T} 4$, compared to the control group who experienced a mean 2.7 (SD3.4) falls. The negative binomial model indicated no significant difference between the groups: incidence rate ratio $0.605,95 \%$ CI 0.243 to $1.502, p=0.3$.

The effect size from T1 to T2 appeared to favour the intervention on the number of falls (Cohen's $d=0.4$; 95\% CI -0.2 to 0.1 ). This effect size was not maintained and reduced to no effect at $\mathrm{T} 3$ and $\mathrm{T} 4$.

\section{Time to first fall}

Participants allocated to the intervention had a median falls-free survival time (time to first fall) of 91 days (range 42 days to 257 days). This was longer than those in the control group who had a median time to first fall of 64 days (range 2 days to 160 days). Time to first fall did not differ significantly between the groups (Log Rank test [Mantel Cox] $\left.X^{2}(1) 0.67, p=0.41\right)$. These data indicate that those in the intervention group were not placed at higher risk of experiencing a fall compared to the control group as a result of the intervention.

\section{Secondary outcomes}

Table 4 shows the means and effect sizes for all secondary outcomes in the domains of balance, functional ability, strength, quality of life, activities of daily living, falls self-efficacy, and foot problems.

\section{Feasibility of outcome data collection}

Collecting data on the number of falls was straightforward since all care homes kept a record of fall events as

Table 3 Mean number (range) of falls per participant and effect size by time point

\begin{tabular}{|c|c|c|c|c|c|}
\hline Outcome measure & Time point & $\begin{array}{l}\text { Podiatry intervention } \\
\text { group }(n=23)\end{array}$ & Control group $(n=20)$ & $p$-value $(95 \% \mathrm{Cl})$ & $\begin{array}{l}\text { Standardised effect } \\
\text { size }^{a} \text { Cohen's d }(95 \% \mathrm{Cl})\end{array}$ \\
\hline \multirow[t]{3}{*}{ Mean (range) falls per participant } & T1-T2 & $0.64(0-3)$ & $1.18(0-6)$ & $0.08(-1.39$ to .31$)$ & $0.4(-0.2$ to 1.0$)$ \\
\hline & $\mathrm{T} 2-\mathrm{T} 3$ & $0.99(0-5)$ & $1.05(0-3)$ & $0.18(-0.88$ to .82$)$ & $0.0(-0.5$ to 0.6$)$ \\
\hline & T3-T4 & $0.77(0-7)$ & $0.83(0-6)$ & $0.47(-0.82$ to 1.11$)$ & $0.0(-0.5$ to 0.6$)$ \\
\hline
\end{tabular}

T1 Baseline, T2 Follow up at end of intervention, T3 Follow up 3 months after end of intervention, T4 Follow up 6 months after end of intervention a Derived from the difference in means of the 2 groups; positive value favours intervention 


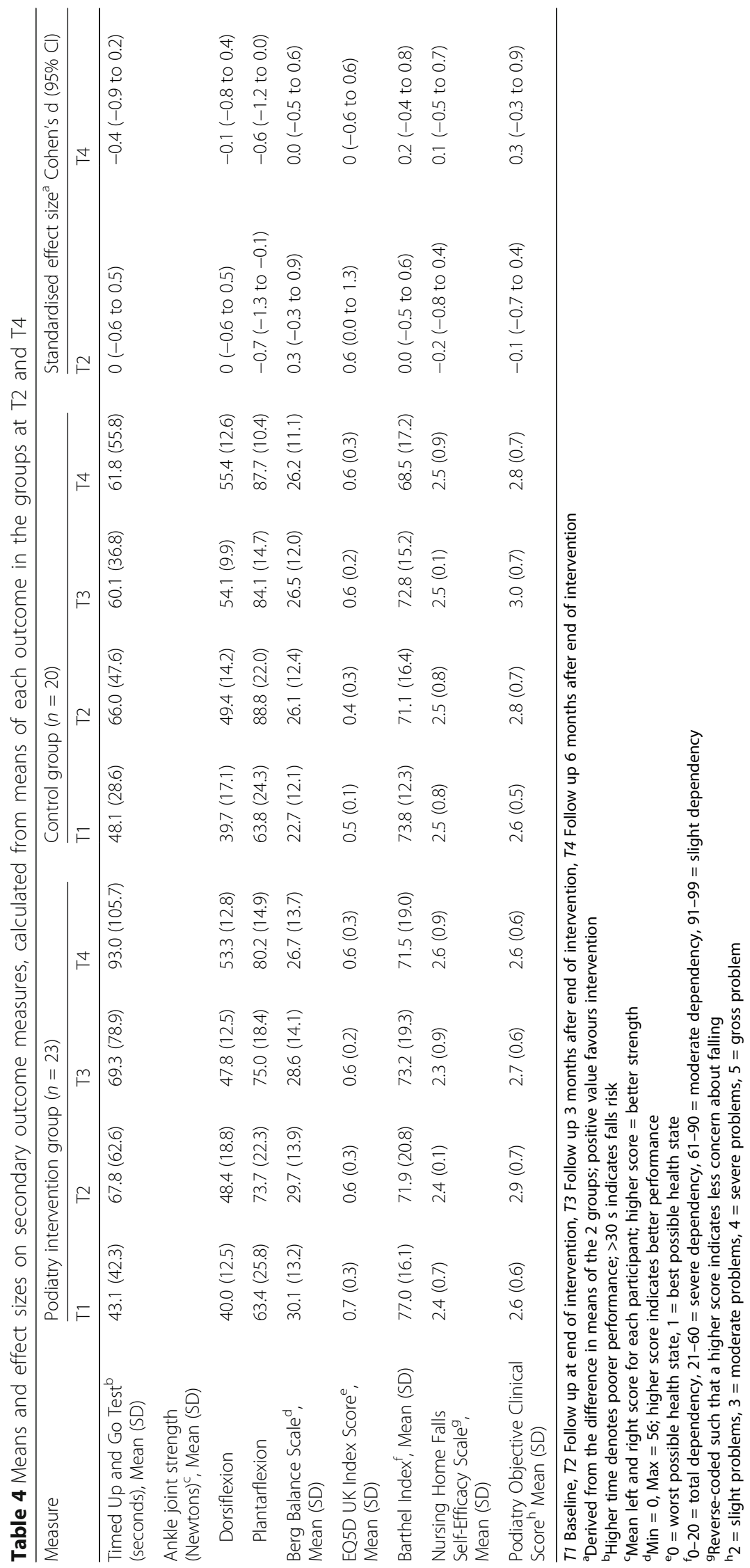


part of their normal documentation processes. Completion of the secondary outcomes was as follows: Podiatry Objective Clinical Score (100\%), Berg Balance Scale (100\%), Timed Up and Go Test (93.4\%), Barthel Index (100\%), EQ-5D (95.3\%), Nursing Home Falls SelfEfficacy Scale (100\%) and ankle strength (98.7\%), 100\% completion was achieved for the Barthel Index. This was because we employed proxy completion of the Barthel Index by care home staff, which would be regarded as normal practice for this measure. Similarly, full completion was achieved for the Podiatry Objective Clinical Score because this relied upon clinician report following examination of the feet. Completion rate of the EQ5D was 95.3\%. The issue preventing full completion was a number of residents having difficulty in understanding how to complete the visual analogue subscale of the EQ5D. Timed Up and Go Test had a completion rate of $93.4 \%$. Dynamometry to measure ankle strength had a completion rate of $98.7 \%$. We obtained data on every item within the Berg Balance Scale, and the Nursing Home Falls Self-Efficacy scale - if residents were unable to complete a particular section they simply received the minimum score possible for that particular manoeuvre or domain, as per the guidance for these measures.

\section{Adverse events and blinding}

No adverse events related to the intervention were reported. The rater maintained a diary of visits throughout the follow up period, and reported 11 instances of unblinding.

\section{Adherence}

Documented exercise adherence (defined by the percentage of participants who reported completing the exercise programme three times per week) was low (35\%). Insole adherence was $48 \%$. Thirteen of the 23 residents in the intervention group were assessed as having sub-optimal footwear, and subsequently received the footwear component of the intervention. All reported wearing these all or most of the time.

\section{Discussion}

The purpose of this study was to assess the feasibility and the potential efficacy of a podiatry intervention to reduce falls in care home residents. We have shown that conducting an RCT of a podiatry intervention to reduce falls in care homes is feasible with regard to recruitment, retention, and intervention delivery. Since this was a pilot trial with a small sample size [40], the effectiveness of the intervention cannot be determined from our results. However the data reported here support testing the intervention in a definitive full scale multicentre trial to test effectiveness.
There were some notable differences in baseline characteristics between the two groups. The majority of the participants were female, however this is representative of the general care home population [45]. Furthermore, there were differences in baseline characteristics in the domains of eye, problems and history of stroke. This is most likely due to our small sample, and in a large trial, we would not anticipate such differences. A small proportion of the screened population of residents $(9.9 \%)$ were eligible to participate in this study because inclusion was restricted to those who were able to provide informed consent. It is well recognised that recruitment problems are compounded in care home research by multi-morbidity and cognitive impairment [20] and this was the experience of recruitment in the present study. However, despite the low recruitment rate, we exceeded our recruitment target, but needed to screen 474 care home residents to achieve this figure (target $=32$; actually recruited $=43$ ). Therefore we have shown that recruitment problems are not insurmountable. It is important to note that the proportion of screened residents who were eligible is essentially a point prevalence figure that met our criteria. It is possible that in a larger study conducted with a much longer recruitment window, a much higher proportion of the population would have met the criteria, and been recruited to the study. Our experience has taught us a number of factors related to care home staff that may have assisted recruitment. First, we relied upon the knowledge of staff to identify residents who they felt would be able to provide informed consent. Second, we reinforced the study as valuable and of importance to their residents. Third, we were sensitive to the care home culture, its organisation, and the level of disruption that the study may have caused.

There was a difference in retention rates between the two arms of the trial, with the control group demonstrating higher attrition. Death, declining health, and no desire to continue with the trial, were the main reasons for this attrition in the control group, and in a small sample such as ours, this difference is not unexpected, particularly given the challenges of retention in studies involving older adults [46]. Our retention rate is lower than pilot trials of falls interventions directed at community dwelling older people (92\%) [47], and community dwelling older people with dementia (91\%) [48] Moreover, our retention rate was lower than pilot trials in care homes not specifically directed at falls, for example mobility training interventions (97\%) [49] and incontinence interventions (91\%) [50]. However, it should be remembered that the follow up period in our study was considerably longer (6 months) than these studies. Our retention rate was similar to the rate reported in a recent feasibility RCT of a falls intervention in care homes [8] and those reported in a number of other full scale 
care trials of care home falls interventions [51, 52]. This is both encouraging and contrary to messages about challenging retention in care home studies [20]. Engaging care home staff to assist with recruitment is an approach we would endorse. Furthermore, to enhance recruitment for a full trial, we would consider alternative design approaches such as cluster randomisation; this approach was not taken in this pilot RCT because the larger sample size requirement and the inherent cost and workload implications of a clustering would have been more costly than a pilot RCT would merit [53].

Our data suggested an effect size that appeared to favour the intervention, but only directly after intervention delivery (T2); this effect disappeared (Cohen's $d=0$ ) at 6-month follow up (T4). These data suggest that if indeed there is any protective effect of the intervention, it reduces rapidly. For any sustained effect of the intervention, care home residents may need to persist with the intervention components on a regular basis. We do not know if, and to what extent, residents continued with the intervention after the end of the protocoled 3-month intervention period, although the reduction in effect size over time suggests that they may not. Our study suggested a trend towards longer falls free survival time in the intervention group compared to the control group, however this measure is not intended to be indicative of any potential benefit of the intervention, rather it was included as a safety measure to ensure that those receiving the intervention were not placed at a higher risk of experiencing a fall as a result of the intervention [28]. Although there are doubts about the validity of applying multiple imputation to missing data in small sample sizes, we felt it was appropriate to do so in this case because: 1 ) it is not acceptable to ignore missing values and the reason they arose [54]; 2) whether imputation is used or not, our statistical analysis needs to be treated with caution, 3) We considered this was the best way to get as accurate an estimate of potential effect as possible [54].

With regard to the secondary outcomes, the differences between the groups were varied, and in some cases contradictory. Despite apparent improvements in balance (Berg balance scale), performance in functional ability (Timed Up and Go Test) worsened both between and within the groups. A possible explanation for this could be declining overall health of our sample. However, the results in the secondary outcomes should be interpreted with caution since we conducted a large number of tests in a small sample. Therefore, as would be expected there was a high degree of variability. Furthermore, in our analyses we did not adjust for multiple testing and the probability that these findings were due to chance cannot be excluded. There were no other important differences between the groups on secondary outcomes and in a future definitive RCT, we would recommend a reduction in the number of secondary outcome measures. This would reduce the burden on participants, and focus interest on outcomes that are most meaningful for residents of care homes, i.e. fallsfree mobility and quality of life [55].

Monitoring adherence to the exercise component of the intervention was a challenge. We recorded $35 \%$ of participants completing the exercise programme in full. As a result of low rates of diary completion it is difficult to determine to what extent participants adhered to the intervention. We would use the term, 'documented adherence, since the results (falls reduction at T2, and increased ankle joint muscle strength) would suggest that residents were completing the exercises more than they were actually documenting. This raises important issues about how adherence to interventions such as this is measured. To monitor adherence, PIRFECT relied upon self-report methods. This approach is fast, inexpensive and easy to implement. Conversely, this approach has poor reliability and validity because of response biases such as acquiescence, social factors such as perceived consequences, and psychological factors such as memory, cognitive ability, and health beliefs [56]. Accurately measuring adherence in exercise interventions is a problem that remains to be solved, although real-time data collection using hand-held mobile devices may hold promise [56]. In addition, the use of accelerometers may be considered in measuring adherence to exercises, however, their use is limited in large clinical groups because they are costly, and require the participant to consistently wear the device, and are not sensitive enough to assess individual limb exercise. [57].

The present study must be viewed in the context of its limitations. First, given the study's low power and relatively short follow up time, the statistical analysis should be treated with caution. Secondly, because of the nature of this multifaceted intervention, it was impossible to blind participants to their group allocation, nor was it possible identify the contribution of each intervention component to reducing falls in the intervention group. Fourth, within care homes, residents who are unable to provide informed consent are the rule rather than the exception, and the fact that inclusion was restricted only to those who could provide informed consent limits the external validity of this study. However, in comparison with other care home falls studies, the mean MMSE score of our sample was 21.2 (classified as mild cognitive impairment), which is only slightly higher than a previous care home falls RCT by McMurdo [58] whose sample had a mean MMSE of 19, and lower than Jensen [52] whose sample had a mean score of 24 . It should be remembered that whilst useful as an indicator of cognitive ability, the Mini Mental State Examination score may mislabel residents who can consent as lacking capacity, 
and residents unable to consent as having capacity [59]. Therefore, recruitment for a subsequent larger study will require ethical approval that allows for the inclusion of residents without capacity to consent, and it is important to consider how this may be achieved in a future definitive trial. Our study was carried out in Scotland where current laws do not allow for consent by proxy for those lacking capacity. However, in a future multicentre trial, as well as recruiting in Scotland, we would intend to recruit in sites in England, where consent by proxy is permissible in law. Lastly, whilst we undertook some process evaluation work during the development of the intervention, our approach did not adhere to published frameworks. The importance of process evaluations has long been recognised, and there is now published guidance on the practical steps for its conduct [60]. Therefore, we will incorporate a formal full process evaluation using this practical guidance in a future trial.

Intervention cost and cost effectiveness is an important consideration. Cost effectiveness could not be determined from a small pilot study, however we can make some remarks about the potential overall cost of the intervention. Purchase of the footwear and orthoses components of the intervention are likely to be the most expensive aspects. However, whoever bears this cost will be dependent upon the health service delivery context in which intervention is delivered. The cost of footwear could conceivably be borne by the resident in some cases. In terms of administering the intervention, apart from initial training from a podiatrist, the exercises are embedded into the normal care home routines and are delivered by care home staff, thus incurring minimal extra cost for health services. The footwear and orthoses aspects of the intervention can be delivered in two visits, with one visit for assessment, and one visit for fitting.

\section{Conclusion}

Delivering and testing a podiatry intervention to reduce care home falls is feasible, despite the numerous challenges that care home research poses. The intervention and trial was also deliverable in care homes of differing sizes and models of ownership (state-owned, privatelyowned). This pilot study not only provides essential information to enable the design and implementation of a full-scale RCT of the intervention, but is also likely to inform other future studies using complex intervention development frameworks with the care home population. The intervention itself is low-cost, easy to implement and uses skills that are standard to podiatrists internationally. Overall, our data support progression to a full multi-centre RCT that incorporates a full process evaluation.

\section{Additional file}

Additional file 1: DVD media that was developed as part of the intervention delivery training programme supplied to the care home staff during the study. (M4 V $53690 \mathrm{~kb}$ )

\section{Abbreviations}

BBS: Berg Balance Scale; EQ-5D: Euroqol five dimensions questionnaire; MMSE: Mini Mental State Exam; MRC: Medical Research Council; NHFSS: Nursing Home Falls Self-Efficacy Scale; NHS: National Health Service; POCS: Podiatry Objective Clinical Score; RCT: Randomised controlled trial; UK: United Kingdom; UKCRC: United Kingdom Clinical Research Collaboration

\section{Acknowledgements}

We gratefully acknowledge the time, effort and commitment to the study from the care home residents, staff and managers - without them, this study could not have happened. We would also like to acknowledge the generous support given to us in terms of recruiting care homes to the study from

Edith Macintosh of the Scottish Care Inspectorate, and Carolyn Wilson who is NHS Tayside's Falls service manager. Dr. Roberta Littleford and Eva Lahnsteiner and their all their colleagues at Tayside Clinical Trials Unit provided invaluable trial management, web support, administrative support and database expertise throughout the duration of the study. Finally, we would like to thank and acknowledge the Hylton Menz and Martin Spink for agreeing to us using and adapting the intervention initially developed by them, and applying it to the care home context.

\section{Funding}

This study was funded by the Chief Scientist Office, Scottish Government Health Department (reference: $\mathrm{CZH/4/701).}$

\section{Availability of data and materials}

Data are available on request from the authors.

\section{Authors' contributions}

GW conceived and designed the study, wrote the funding application, study protocol, conducted baseline data collection, conducted the statistical analysis, and wrote the manuscript. HM conceived the study and the original intervention upon which this work is based, assisted with statistical analysis, contributed methodological advice throughout, and contributed to the final manuscript. SM conducted and coordinated follow-up data collection and data management. SO dispensed statistical and methodological advice throughout the study, contributed to the final data analysis, and revised the statistical content of the manuscript. FS and BW contributed substantial methodological and intellectual input throughout the study. ZY conducted baseline data collection, provided methodological input, and contributed to day-to-day management of the study. JM conceived and designed the study, wrote the funding application and provided methodological and intellectual advice throughout. All Authors read and approved the final manuscript.

\section{Ethics approval and consent to participate}

Ethics approval for this study was sought and obtained from the East of Scotland Research Ethics Service (EOSRES), reference number ES/12/0088. The decision to recruit was based on the ability of the prospective participant to explain back to the research podiatrist what was required by their participation in the study. Written consent to take part was obtained form each participant.

\section{Consent for publication \\ Not applicable.}

\section{Competing interests}

The authors declare that they have no competing interests.

\section{Publisher's Note}

Springer Nature remains neutral with regard to jurisdictional claims in published maps and institutional affiliations. 


\section{Author details}

${ }^{1} \mathrm{NHS}$ Tayside, Ageing and Health, and School of Nursing \& Health Sciences, University of Dundee, Dundee, UK. ${ }^{2}$ School of Allied Health, College of Science, Health and Engineering, La Trobe University, Victoria, Australia. ${ }^{3} \mathrm{NHS}$ Tayside and Nursing \& Midwifery Research Unit, Glasgow Caledonian University, Glasgow, UK. ${ }^{4}$ School of Medicine, University of Dundee, Dundee, UK. ${ }^{5}$ School of Medicine, University of St Andrews, St Andrews, UK. ${ }^{6}$ School of Health and Social Care, Napier University, Edinburgh, UK. ${ }^{7} \mathrm{NHS}$ Tayside, Perth, UK. ${ }^{8}$ School of Nursing and Health Sciences, University of Dundee, Dundee, UK.

Received: 18 April 2017 Accepted: 6 July 2017

Published online: 12 July 2017

\section{References}

1. Tinetti ME, Speechley M. Prevention of falls among the elderly. N Engl J Med. 1989;320:1055-9.

2. Dyer CAE, Taylor GJ, Reed M, Dyer CA, Robertson DR, Harrington R. Falls prevention in residential care homes: a randomised controlled trial. Age Ageing. 2004;33:596-602.

3. Rapp K, Becker C, Cameron ID, König H-H, Büchele G. Epidemiology of falls in residential aged care: analysis of more than 70,000 falls from residents of Bavarian nursing homes. J Am Med Dir Assoc. 2012;13(187):e1-6.

4. Gordon AL, Franklin M, Bradshaw L, Logan P, Elliott R, Gladman JRF. Health status of UK care home residents: a cohort study. Age Ageing. 2014;43:97-103.

5. Close JCT. Prevention of falls in older people. Disabil Rehabil. 2005;27:1061-71.

6. Tinetti ME, Williams CS. Falls, injuries due to falls, and the risk of admission to a nursing home. N Engl J Med. 1997;337:1279-84

7. Cameron ID, Gillespie LD, Robertson MC, Murray GR, Hill KD, Cumming RG, et al. Interventions for preventing falls in older people in care facilities and hospitals. Cochrane Database Syst Rev. 2012;12:CD005465.

8. Walker GM, Armstrong S, Gordon AL, Gladman J, Robertson K, Ward M, et al. The falls in care home study: a feasibility randomized controlled trial of the use of a risk assessment and decision support tool to prevent falls in care homes. Clin Rehabil. 2016;30:972-83.

9. Ambrose AF, Paul G, Hausdorff JM. Risk factors for falls among older adults: a review of the literature. Maturitas. 2013;75:51-61.

10. Dunn JE, Link CL, Felson DT, Crincoli MG, Keysor JJ, Mckinlay JB. Prevalence of foot and ankle conditions in a multiethnic community sample of older adults. Am J Epidemiol. 2004;159:491-8.

11. Griffith L, Raina P, Wu H, Zhu B, Stathokostas L. Population attributable risk for functional disability associated with chronic conditions in Canadian older adults. Age Ageing. 2010;39:738-45.

12. Menz HB, Morris ME, Lord SR. Foot and ankle risk factors for falls in older people: a prospective study. J Gerontol Ser A-BiolSci Med Sci. 2006;61:866-70.

13. Awale A, Hagedorn TJ, Dufour AB, Menz HB, Casey VA, Hannan MT. Foot function, foot pain, and falls in older adults: the Framingham foot study. Gerontol. 2017;63:1-7.

14. Mickle KJ, Munro BJ, Lord SR, Menz HB, Steele JR. ISB clinical biomechanics award 2009: toe weakness and deformity increase the risk of falls in older people. Clin Biomech. 2009;24:787-91.

15. Sherrington C, Menz HB. An evaluation of footwear worn at the time of fallrelated hip fracture. Age Ageing. 2003;32:310-4.

16. Schwenk M, Jordan ED, Honarvararaghi B, Mohler J, Armstrong DG, Najafi B. Effectiveness of foot and ankle exercise programs on reducing the risk of falling in older adults a systematic review and meta-analysis of randomized controlled trials. J Pod Med Assoc. 2013;103:534-47.

17. Hatton AL, Rome K, Dixon J, Martin DJ, McKeon PO. Footwear interventions: a review of their sensorimotor and mechanical effects on balance performance and gait in older adults. J Am Podiatr Med Assoc. 2013;103:516-33.

18. Spink MJ, Menz HB, Fotoohabadi MR, Wee E, Landorf KB, Hill KD, et al. Effectiveness of a multifaceted podiatry intervention to prevent falls in community dwelling older people with disabling foot pain: randomised controlled trial. BMJ. 2011;342:d3411.

19. Cockayne S, Adamson J, Clarke A, Corbacho B, Fairhurst C, Green L, et al. Cohort randomised controlled trial of a multifaceted podiatry intervention for the prevention of falls in older people (the REFORM trial). PLoS One. 2017;12:e0168712.

20. Hall S, Longhurst S, Higginson IJ. Challenges to conducting research with older people living in nursing homes. BMC Geriatr. 2009;9:38.
21. Garcia C, Kelley CM, Dyck MJ. Nursing home recruitment: trials, tribulations, and successes. Appl Nurs Res. 2013:26:1-3.

22. Craig P, Dieppe P, Macintyre S, Michie S, Nazareth I, Petticrew M. Developing and evaluating complex interventions: the new Medical Research Council guidance. BMJ. 2008;337:a1655.

23. Wylie G, Young Z, Littleford R, Sullivan F, Coyle J, Williams B, et al. Finding your feet : the development of a podiatry intervention to reduce falls in care home residents. J Foot Ankle Res BioMed Cen Ltd. 2015;8:A7.

24. Wylie G, Young Z, Littleford R, Sullivan F, Williams B, Menz H, et al. Reflecting on the methodological challenge of recruiting older care home residents to podiatry research. J. Foot Ankle Res. BioMed Cen Ltd. 2015;8:A10.

25. Tromp AM, Pluijm SMF, Smit JH, Deeg DJH, Bouter LM, Lips P. Fall-risk screening test: a prospective study on predictors for falls in community dwelling elderly. J Clin Epidemiol. 2001;54:837-44.

26. HCPC. Health and Care Professions Council [Internet]. [cited 2016 Dec 6]. Available from: http://www.hcpc-uk.org/aboutregistration/professions/index. asp?id=3\#profDetails.

27. Whelan PJP, Oleszek J, Macdonald A, Gaughran F. The utility of the minimental state examination in guiding assessment of capacity to consent to research. Int Psychogeriatr. 2009;21:338-44.

28. Lamb SE, Jørstad-Stein EC, Hauer K, Becker C. Development of a common outcome data set for fall injury prevention trials: the prevention of falls network Europe consensus. J Am Geriatr Soc. 2005;53:1618-22.

29. Fleming J, Matthews FE, Brayne C. Falls in advanced old age: recalled falls and prospective follow-up of over-90-year-olds in the Cambridge City over75s cohort study. BMC Geriatr. 2008;8:6.

30. Farndon L, Barnes A, Littlewood K, Harle J, Beecroft C, Burnside J, et al. Clinical audit of core podiatry treatment in the NHS. J Foot Ankle Res. 2009;2:7.

31. Christofoletti G, Oliani MM, Gobbi S, Stella F, Bucken Gobbi LT, Renato CP. A controlled clinical trial on the effects of motor intervention on balance and cognition in institutionalized elderly patients with dementia. Clin Rehabil. 2008;22:618-26.

32. Nordin E, Rosendahl E. Timed 'up \& go' test: reliability in older people dependent in activities of daily living — focus on cognitive state. Phys Ther. 2006;86:646

33. Shah S, Vanclay F, Cooper B. Improving the sensitivity of the Barthel index for stroke rehabilitation. J Clin Epidemiol. 1989;42:703-9.

34. Kunz S. Psychometric properties of the EQ-5D in a study of people with mild to moderate dementia. Qual Life Res. 2010;19:425-34.

35. Lach HW, Ball LJ, Birge SJ. The nursing home falls self-efficacy scale: development and testing. Clin Nurs Res. 2012;21:79-91.

36. Spink MJ, Fotoohabadi MR, Menz HB. Foot and ankle strength assessment using hand-held dynamometry: reliability and age-related differences. Gerontol. 2010;56:525-32.

37. Aboutorabi A, Bahramizadeh M, Arazpour M, Fadayevatan R, Farahmand F, Curran S, et al. A systematic review of the effect of foot orthoses and shoe characteristics on balance in healthy older subjects. Prosthetics Orthot Int. 2016:40:170-81.

38. Menz HB, Sherrington C. The footwear assessment form: a reliable clinical tool to assess footwear characteristics of relevance to postural stability in older adults. Clin Rehabil. 2000;14:657-64.

39. Ribeiro F, Teixeira F, Brochado G, Oliveira J. Impact of low cost strength training of dorsi- and plantar flexors on balance and functional mobility in institutionalized elderly people. Geriatr Gerontol Int. 2009;9:75-80.

40. Arain M, Campbell MJ, Cooper CL, Lancaster GA. What is a pilot or feasibility study? A review of current practice and editorial policy. BMC Med Res Methodol. 2010;10:67.

41. Fritz CO, Morris PE, Richler JJ. Effect size estimates: current use, calculations, and interpretation. J Exp Psychol Gen. 2012;141:2-18.

42. Ullah S, Finch CF, Day L. Statistical modelling for falls count data. Accid Anal Prev. 2010;42:384-92.

43. Lamb SE, Jorstad-Stein EC, Hauer K, Becker C, PFNE. Development of a common outcome data set for fall injury prevention trials: the prevention of falls network Europe consensus. J Am Geriatr Soc. 2005: 53:1618-22.

44. Boutron I, Moher D, Altman DG, Schulz KF, Ravaud P. Extending the CONSORT statement to randomized trials of nonpharmacologic treatment: explanation and elaboration. Ann Intern Med. 2008;148:295-309.

45. McMurdo MET, Roberts H, Parker S, Wyatt N, May H, Goodman C, et al. Improving recruitment of older people to research through good practice. Age Ageing. 2011;40:659-65. 
46. Mody L, Miller DK, McGloin JM, Freeman M, Marcantonio ER, Magaziner J, et al. Recruitment and retention of older adults in aging research. J Am Geriatr Soc. 2008;56:2340-8.

47. Barker AL, Talevski J, Bohensky MA, Brand CA, Cameron PA, Morello RT. Feasibility of Pilates exercise to decrease falls risk : A pilot randomized controlled trial in community-dwelling older people. Clin Rehabil. 2015;30:1-13.

48. Wesson J, Clemson L, Brodaty H, Lord S, Taylor M, Gitlin L, et al. A feasibility study and pilot randomised trial of a tailored prevention program to reduce falls in older people with mild dementia. BMC Geriatr. 2013;13:89.

49. Sackley CM, Rodriguez NA, van den Berg M, Badger F, Wright C, Besemer J, et al. A phase II exploratory cluster randomized controlled trial of a group mobility training and staff education intervention to promote urinary continence in UK care homes. Clin Rehabil. 2008;22:714-21.

50. Blekken LE, Nakrem S, Gjeilo KH, Norton C, Morkved S, Vinsnes AG. Feasibility, acceptability, and adherence of two educational programs for care staff concerning nursing home patients' fecal incontinence: a pilot study preceding a cluster-randomized controlled trial. Implement Sci. 2015;10:72.

51. Christofoletti G, Oliani MM, Gobbi S, Stella F. Effects of motor intervention in elderly patients with dementia - an analysis of randomized controlled trials. Geriatr Rehabil. 2007;23:149-54.

52. Jensen J, Nyberg L, Gustafson Y, Lundin-Olsson L. Fall and injury prevention in residential care-effects in residents with higher and lower levels of cognition. J Am Geriatr Soc. 2003;51:627-35.

53. Christie J, O'Halloran P, Stevenson M. Planning a cluster randomized controlled trial. Nurs Res. 2009;58:128-34.

54. Sterne JAC, White IR, Carlin JB, Spratt M, Royston P, Kenward MG, et al Multiple imputation for missing data in epidemiological and clinical research: potential and pitfalls. BMJ. 2009;338:b2393.

55. Burns E, Nair S. New horizons in care home medicine. Age Ageing. 2014;43:2-7.

56. Vitolins MZ, Rand CS, Rapp SR, Ribisl PM, Sevick MA. Measuring adherence to behavioral and medical interventions. Control Clin Trials. 2000;21:188S-94S.

57. Bollen JC, Dean SG, Siegert RJ, Howe TE, Goodwin VA. A systematic review of measures of self-reported adherence to unsupervised home-based rehabilitation exercise programmes, and their psychometric properties. BMJ Open. 2014;4:e005044.

58. McMurdo ME, Millar AM, Daly F. A randomized controlled trial of fall prevention strategies in old peoples' homes. Gerontol. 2000;46:83-7.

59. Whelan PJP, Oleszek J, Macdonald A, Gaughran F. The utility of the Minimental State Examination in guiding assessment of capacity to consent to research. Int Psychogeriatr. 2017;21:338-44.

60. Moore GF, Audrey S, Barker M, Bond L, Bonell C, Hardeman W, et al. Process evaluation of complex interventions: Medical Research Council guidance. BMJ. 2015;350:h1258.

\section{Submit your next manuscript to BioMed Central and we will help you at every step:}

- We accept pre-submission inquiries

- Our selector tool helps you to find the most relevant journal

- We provide round the clock customer support

- Convenient online submission

- Thorough peer review

- Inclusion in PubMed and all major indexing services

- Maximum visibility for your research

Submit your manuscript at www.biomedcentral.com/submit 